\title{
Discrete Rigid Body Dynamics and Optimal Control
}

\author{
Anthony M. Bloch ${ }^{1}$ \\ Department of Mathematics \\ University of Michigan \\ Ann Arbor MI 48109 \\ abloch@math.lsa.umich.edu \\ Jerrold E. Marsden ${ }^{3}$ \\ Control and Dynamical Systems 107-81 \\ California Institute of Technology \\ Pasadena, CA 91125 \\ marsden@cds.caltech.edu
}

\author{
Peter E. Crouch ${ }^{2}$ \\ Center for Systems Science \\ and Engineering \\ Arizona State University \\ Tempe, AZ 85287 \\ peter.crouch@asu.edu \\ Tudor S. Ratiu ${ }^{4}$ \\ Department of Mathematics \\ University of California \\ Santa Cruz, CA 95064 \\ ratiu@cats.ucsc.edu
}

\begin{abstract}
In this paper we analyze an alternative formulation of the rigid body equations, their relationship with the discrete rigid body equations of Moser-Veselov and their formulation as an optimal control problem. In addition we discuss a general class of discrete optimal control problems.
\end{abstract}

Keywords: optimal control, discrete dynamics, rigid body mechanics

\section{Introduction}

The main goal of this paper is to give an alternative formulation of rigid body equations which is based on the use of the maximum principle, to establish its connection with the Moser-Veselov [1991] discrete rigid body equations, and through this, with problems in numerical analysis.

There has been much interest in recent years in structured algorithms (such as symplectic methods) for integrating Hamiltonian systems and, in particular, rigid body mechanics - see for example McLachlan and Scovel [1995], Marsden and Wendlandt [1997], and Marsden, Patrick, and Shkoller [1997] and references therein. A particular feature of the rigid body equations is that the discrete form is still integrable in a precise sense, as shown by Moser and Veselov [1991] (see also Deift, Li, and Tomei [1992]).

\footnotetext{
${ }^{1}$ Research partially supported by National Science Foundation grants DMS-9496221 and DMS-9803181 and AFOSR grant F49620-96-1-0100.

${ }^{2}$ Work supported in part by NSF grant DMS 91011964 and NATO grant CRG 910926

${ }^{3}$ Research partially supported by AFOSR contract F49620-951-0419.

${ }^{4}$ Work supported in part by NSF grant DMS-9503273 and DOE contract DE-FG03-95ER25245-A000
}

0-7803-4394-8/98 \$10.00 (C) 1998 IEEE
Our formulation of the equations will be shown to be useful not only for understanding the discrete rigid body equations, but for understanding integrability on the full phase space (not just the reduced dynamics). Also of interest is understanding the variational formulation of the discrete equations and the link with the smooth case. In our setting, this is reflected by the use of the maximum principle.

Our work is related to the study of optimal control problems on adjoint orbits of Lie groups, defined using the so-called normal metric and a right-invariant generalization of it; see Brockett [1994], Bloch and Crouch [1996], and Bloch, Brockett, and Crouch [1997].

If the flow is generated by the geodesic spray of a bi-invariant metric, we obtain a pair of coupled double bracket equations which are explicitly soluble. These equations are quite different from the double bracket equations discussed in Brockett [1989], Bloch, Brockett, and Ratiu [1990, 1992] and Bloch, Flaschka and Ratiu [1990]. A related paper of interest on explicitly soluble optimal control problems is that of Faybusovich [1988].

Specifically, we study the generalized rigid body equations and show that they arise from coupled double bracket equations and can be written in the form

$$
\begin{aligned}
& \dot{Q}=Q \Omega \\
& \dot{P}=P \Omega
\end{aligned}
$$

where $\Omega=J^{-1} M$ and $M=P Q^{T}-Q P^{T}$. Here, $Q$ denotes the configuration of the body in the body frame, $M$ is the body angular momentum and $J$ is the (body fixed) inertia tensor. We shall also give a discrete version of these equations and show how they arise from a discrete optimal control problem. 


\section{The Geodesic Sprays for Left and Right Invariant Metrics}

In this section we review the classical rigid body equations and a novel alternative formulation. We shall also compare the left and right invariant geodesic sprays, derive the conservation of momentum in each case, and discuss the duality between these equations.

We recall that the rigid body equations on $S O(3)$ (or, generally, on $S O(n)$, or any compact Lie group which is the intersection of the normal and compact real forms of a complex semisimple Lie group-see e.g. Ratiu [1980]) may be written (in the left trivialization of $\left.T^{*} S O(n)\right)$ as

$$
\begin{aligned}
\dot{Q} & =Q \Omega \\
\dot{M} & =[M, \Omega],
\end{aligned}
$$

where $Q \in S O(n)$ denotes the configuration space variables (the attitude of the body), $\Omega \in \mathfrak{s o}(n)$ is the body angular velocity, and $M:=J(\Omega)=\Lambda \Omega+\Omega \Lambda \in \mathfrak{s o}(n)$ is the body angular momentum. Here $J: \mathfrak{s o}(n) \rightarrow \mathfrak{s o}(n)$ is the symmetric positive definite operator defined by $J(\Omega)=\Lambda \Omega+\Omega \Lambda$, where $\Lambda$ is a diagonal matrix satisfying $\Lambda_{i}+\Lambda_{j}>0$ for all $i \neq j$. These equations are the geodesic spray on $T S O(n)$, left trivialized as $S O(n) \times \mathfrak{s o}(n)$, relative to the left invariant metric whose expression at the identity is

$$
\left\langle M_{1}, M_{2}\right\rangle=-\frac{1}{4} \operatorname{trace}\left(M_{1} J^{-1}\left(M_{2}\right)\right)
$$

For $\Omega \in \mathfrak{s o}(n)$, and $Q, P \in S O(n)$, consider the following equations for left invariant vector fields:

$$
\begin{aligned}
& \dot{Q}=Q \Omega \\
& \dot{P}=P \Omega .
\end{aligned}
$$

Proposition 2.1 If $\Omega:=J^{-1}(M)$ and $M=Q^{T} P-$ $P^{T} Q$, then the equations (2.3) imply the rigid body equations (2.1).

Proof Differentiating $M=Q^{T} P-P^{T} Q$ and using the equations (2.3) gives the second of equations (2.1).

For this reason, equations (2.3) are called the symmetric rigid body equations on $S O(n) \times S O(n)$.

Proposition 2.2 For the left invariant geodesic spray (2.1) on $T S O(n)$ (that is, the generalized rigid body equations), the spatial angular momentum is given by $m=P Q^{T}-Q P^{T}$ and it is conserved along the rigid body flow.

Proof Observe that $m$ is obtained from the left invariant body angular momentum $M$ by $m=Q M Q^{T}$ and is thus the spatial angular momentum. Differentiating along (2.3) we find $\dot{m}=0$.
Consider the right invariant Riemannian metric on $S O(n)$ whose value at the identity is given by (2.2). The geodesic spray of this metric on $T S O(n)$, right trivialized as $S O(n) \times \mathfrak{s o}(n)$, is given by

$$
\begin{aligned}
\dot{Q} & =\omega Q \\
\dot{m} & =[\omega, m] .
\end{aligned}
$$

For $\omega \in \mathfrak{s o}(n)$, consider the right invariant vector fields

$$
\begin{aligned}
& \dot{Q}=\omega Q \\
& \dot{P}=\omega P .
\end{aligned}
$$

As before, it is easy to check that

Proposition 2.3 If $\omega:=J^{-1}(m)$ and $m=P Q^{T}-$ $Q P^{T}$, then the equations (2.5) imply the geodesic spray equations (2.4).

Equations (2.5) are thus called the right invariant symmetric rigid body equations on $S O(n) \times S O(n)$. In this case it follows that the body angular momentum is $M:=Q^{T} m Q=Q^{T} P-P^{T} Q$ and that it is conserved along the flow of (2.4).

Given the geodesic spray (2.4) of the right invariant metric on $S O(n)$, we may solve (in a neighborhood of $m=0)$ for the variable $P$ in the expression

$$
m=P Q^{T}-Q P^{T}
$$

Locally,

$$
P=\left(e^{\sinh ^{-1} m / 2}\right) Q
$$

since around $m=0$ we have

$$
m=e^{\sinh ^{-1} m / 2}-e^{-\sinh ^{-1} m / 2} .
$$

For $\mathfrak{s o}(n)$ however, sinh is many to one, so the two representations (2.5) and (2.6) are not entirely equivalent. A similar calculation may be done in the left invariant case.

\section{Optimal Control}

We begin this section by noting the following result obtained by Bloch and Crouch [1996].

Proposition 3.1 The optimal control problem

$$
\min _{u \in \mathfrak{s o}(n)} \frac{1}{4} \int\langle u, J(u)\rangle d t
$$

subject to $\dot{Q}=u Q$, yields the equations (2.5).

The optimal controls in this case are given by

$$
u=J^{-1}\left(P Q^{T}-Q P^{T}\right)
$$


There is a similar result for the left invariant case. We now show how both the left and right invariant symmetric rigid body equations arise from a rather general optimal control problem that includes the one above as a special case (see Bloch and Crouch [1996]). $U(n)$

Let $\mathbf{u}(n)$ denote the Lie algebra of the unitary group

Theorem 3.2 Let $Q$ be a $p \times q$ complex matrix and let $u \in \mathfrak{u}(p)$ and $V \in \mathfrak{u}(q)$. Let $J_{u}$ and $J_{V}$ be constant symmetric positive definite operators on the space of complex $p \times p$ and $q \times q$ matrices respectively and let $\langle\cdot, \cdot\rangle$ denote the trace inner product $\langle A, B\rangle=\operatorname{trace} A^{*} B$, where $A^{*}$ is the adjoint (transpose conjugate).

Consider the optimal control problem over $\mathfrak{u}(p) \times$ $\mathfrak{u}(q)$

$$
\min _{u, V} \frac{1}{4} \int\left\{\left\langle u, J_{u} u\right\rangle+\left\langle V, J_{V} V\right\rangle\right\} d t
$$

subject to

$$
\dot{Q}=u Q-Q V
$$

Then the optimal controls are given by

$$
\begin{aligned}
u & =J_{u}^{-1}\left(P Q^{*}-Q P^{*}\right) \\
V & =J_{V}^{-1}\left(P^{*} Q-Q^{*} P\right) .
\end{aligned}
$$

and the optimal evolution of the states $Q$ and costates $P$ is given by

$$
\begin{aligned}
& \dot{Q}=J_{u}^{-1}\left(P Q^{*}-Q P^{*}\right) Q-Q J_{V}^{-1}\left(P^{*} Q-Q^{*} P\right) \\
& \dot{P}=J_{u}^{-1}\left(P Q^{*}-Q P^{*}\right) P-P J_{V}^{-1}\left(P^{*} Q-Q^{*} P\right) .
\end{aligned}
$$

We remark that this result does not preclude the existence of conjugate points.

Note also that $J_{u}$ and $J_{V}$ are in general different operators acting on different spaces. In certain case (see the rigid body below) the spaces and the operators may be taken to be the same.

We have the immediate corollary:

Corollary 3.3 For $J_{u}$ and $J_{V}$ equal to the identity, the optimal control equations for the problem (3.3) subject to (3.4) are

$$
\begin{aligned}
& \dot{Q}=P Q^{*} Q+Q Q^{*} P-2 Q P^{*} Q \\
& \dot{P}=2 P Q^{*} P-Q P^{*} P-P P^{*} Q
\end{aligned}
$$

Further, in the general case we have

Corollary 3.4 The equations (3.6) are given by the double double bracket equations

$$
\begin{aligned}
& \dot{\hat{Q}}=\left[\hat{Q}, \hat{J}^{-1}[\hat{P}, \hat{Q}]\right] \\
& \dot{\hat{P}}=\left[\hat{P}, \hat{J}^{-1}[\hat{P}, \hat{Q}]\right]
\end{aligned}
$$

where $\hat{J}$ is the operator $\operatorname{diag}\left(J_{u}, J_{V}\right)$,

$$
\hat{Q}=\left[\begin{array}{ll}
0 & Q \\
-Q^{*} & 0
\end{array}\right] \in \mathfrak{u}(p+q),
$$

$Q$ is a complex (real) $p \times q$ matrix of full rank, $Q^{*}$ is its adjoint, and similarly for $P$.

This problem was motivated by optimal control problem on adjoint orbits of compact Lie groups as discussed in Brockett [1994].

Let $\mathfrak{g}$ be a complex semisimple Lie algebra, $\mathfrak{k}$ its compact real form, and $K$ the corresponding compact group. In this case a natural drift free control system on an adjoint orbit of $K$ takes the form

$$
\dot{x}=[x, u]
$$

for $x, u \in \mathfrak{k}$. Then we may consider the following generalization of the functional suggested by Brockett [1994]

$$
\eta(x, u)=\int_{0}^{t_{f}}\left(\frac{1}{2}\|u\|^{2}-V(x)\right) d t
$$

where $\|\cdot\|=\langle\cdot, \cdot\rangle^{1 / 2}$ is the norm induced on $k$ by the negative of the restriction of the Killing form $\kappa(\cdot, \cdot)$ on $\mathfrak{g}$ to $\mathfrak{k}$ and $V$ is a smooth function on $\mathfrak{k}$. The pairing between vectors $x$ in $\mathfrak{g}$ and dual vectors $p$ in $\mathfrak{g}^{*}$ is written $\langle p, x\rangle=-\kappa(x, p)$. For details see Bloch, Brockett and Crouch [1997].

This general optimal control problem gives the geodesic flow on complex and real Grassmannians of $q$ planes in $(n+1)$-space $G_{q, n+1}(\mathbb{C})$ or $G_{q, n+1}(\mathbb{R})$.

The complex Grassmannian is given by

$$
U(n+1) / U(q) \times U(p), \quad q+p=n+1, q \leq p
$$

and the real Grassmannian by

$$
S O(n+1) / S O(q) \times S O(p), q+p=n+1, q \leq p
$$

where $U(n)$ is the unitary group and $S O(n)$ the special orthogonal group. In either case let $\mathfrak{k}=\mathfrak{k}_{0} \oplus \mathfrak{m}$ be the vector space direct sum decomposition corresponding to $K / K_{0}\left(K=U(n+1)\right.$ or $S O(n+1)$ and $K_{0}=U(q) \times$ $U(p)$ or $S O(q) \times S O(p))$. We may thus represent a point in the complex (resp. real) Grassmannian by a matrix

$$
\hat{Q}=\left[\begin{array}{ll}
0 & Q \\
-Q^{*} & 0
\end{array}\right] \in \mathfrak{m},
$$

where $Q$ is a complex (resp. real) $p \times q$ matrix of full rank and $Q^{*}$ is its adjoint. A point in $\mathfrak{k}_{0}$ may be represented by the matrix

$$
\hat{K}=\left[\begin{array}{ll}
K_{1} & O \\
O & K_{2}
\end{array}\right]
$$

where $K_{1} \in \mathfrak{u}(p)$ (resp. $\mathfrak{s o}(p)$ ) and $K_{2} \in \mathfrak{u}(q)$ (resp. $\mathfrak{s o}(q))$. Define $\hat{P}$ to be a similarly partitioned matrix. 
Then tangent vecto:s to the Grassmannian may be represented by matrices of the form $[\hat{Q}, \hat{K}]$.

Since tangent vectors are given by brackets, just as in the case of orbits, a normal metric may be defined and the geodesic equations on the real or complex Grassmannian are given by (see Bloch, Brockett, and Crouch [1997])

$$
\begin{aligned}
& \dot{\hat{Q}}=[\hat{Q},[\hat{P}, \hat{Q}]] \\
& \dot{\hat{P}}=[\hat{P},[\hat{P}, \hat{Q}]],
\end{aligned}
$$

where $\hat{Q}$ is given by (3.9) and similarly for $\hat{P}$.

In fact the formalism developed here can be combined with the work of Thimm [1981] to give an explicit proof of complete integrability of the geodesic flow on symmetric spaces such as the real and complex Grassmannians. In particular, it is possible to derive explicitly a complete set of commuting flows and to prove their involutivity. This is the subject of Bloch, Brockett and Crouch [1997]. We consider the case of integrability of the rigid body below.

The rigid body equations may be given as a singular case of the double double bracket equations discussed earlier for the general optimal control problem. Let

$$
\hat{Q}=\left[\begin{array}{ll}
0 & Q \\
-Q^{T} & 0
\end{array}\right]
$$

as before and similarly for $\hat{P}$, where these matrices are now taken to lie in $\mathfrak{s o}(2 n)$ with the nonzero blocks in $S O(n)$.

Then we get both the left and right symmetric rigid body equations simultaneously from our general optimal control problem as follows:

Corollary 3.5 The symmetric rigid body equations on $S O(n) \times S O(n)$ are given by the double double bracket equations (3.6) or (3.8) if $Q, P \in S O(n)$. To obtain the equations in their left invariant form set $J_{V}=J$ and $J_{u}^{-1}=0$. To obtain the equations in their right invariant form set $J_{u}=J$ and $J_{V}^{-1}=0$.

One sees that the equations (3.6) are literally the sum of the left and right invariant symmetric rigid body equations.

\section{Moser-Veselov Discretization}

We recall now the Moser-Veselov [1991] discrete rigid body equations. Discretize the configuration matrix and set

$$
\begin{aligned}
\Omega_{k} & =Q_{k}^{T} Q_{k-1} \\
M_{k} & =\Omega_{k}^{T} \Lambda-\Lambda \Omega_{k} .
\end{aligned}
$$

Then the Moser-Veselov discrete rigid body equations are given by:

$$
M_{k+1}=\Omega_{k} M_{k} \Omega_{k}^{T}
$$

These equations can be obtained by a discrete variational principle (see Moser and Veselov [1991]): one considers the stationary points of the functional

$$
S=\sum_{k} \operatorname{Tr}\left(Q_{k} \Lambda Q_{k+1}^{T}\right)
$$

on sequences of orthogonal $n \times n$ matrices. The stationary points of this functional (with fixed endpoints) are easily obtained by the use of Lagrange multipliers.

We now show how to exhibit these equations in the spirit of our left invariant symmetric rigid body equations. Set

$$
M_{k}=Q_{k-1}^{T} P_{k}-P_{k}^{T} Q_{k-1}
$$

and similarly set

$$
m_{k}=P_{k} Q_{k-1}^{T}-Q_{k-1} P_{k}^{T} .
$$

Then

$$
M_{k+1}=Q_{k}^{T} P_{k+1}-P_{k+1} Q_{k}=Q_{k}^{T} m_{k+1} Q_{k}
$$

Since $m$ is conserved, this equals

$$
Q_{k}^{T} m_{k} Q_{k}=Q_{k}^{T} Q_{k-1} M_{k} Q_{k-1}^{T} Q_{k}
$$

Setting now $\Omega_{k}=Q_{k}^{T} Q_{k-1}$ we obtain $M_{k+1}=$ $\Omega_{k} M_{k} \Omega_{k}^{T}$ and thus we have precisely the Moser-Veselov equations.

One can consider in general discrete versions of mechanical systems. A key notion is that of the discrete Lagrangian which is a map $\mathbb{L}: Q \times Q \rightarrow \mathbb{R}$. The important point here is that the velocity phase space $T Q$ of Lagrangian mechanics is replaced by $Q \times Q$. What is particularly interesting about our smooth version of the rigid body equations is that the covering equations are naturally on $Q \times Q$, even in this smooth setting.

In the discrete setting, the action integral of Lagrangian mechanics is replaced by an action sum $\mathbb{S}=$ $\sum_{k} \mathbb{L}\left(Q_{k+1}, Q_{k}\right)$ where $Q_{k} \in Q$, the sum is over discrete time, and the equations are obtained by a discrete action principle which minimizes the discrete action.

Mechanical integrators derived from this approach conserve momentum and are symplectic. Remarkably, methods of this type can even be used to integrate PDE's (see Marsden, Patrick and Shkoller [1997]).

\section{Discrete Optimal Control Problems}

One can obtain the Moser Veselov equations as a special case of a general class of discrete optimal control equations. We state some results here - the proofs will appear in a forthcoming publication.

Let $\Omega$ denote a suitable class of control functions and let $\langle\rangle=,a^{T} b$ denote a pairing between vectors. 
Proposition 5.1 The optimal control problem

$$
\min _{u_{k}} \sum_{k=0}^{N} g\left(x_{k}, u_{k}\right)
$$

subject to $x_{k+1}=f\left(x_{k}, u_{k}\right)$ for $u_{k} \in \Omega$, yields the optimal control equations

$$
\begin{aligned}
p_{k} & =\frac{\partial H}{\partial x_{k}}\left(p_{k+1}, x_{k}, u_{k}^{*}\right)^{T} \\
x_{k+1} & =\frac{\partial H}{\partial p_{k+1}}\left(p_{k+1}, x_{k}, u_{k}^{*}\right)^{T}
\end{aligned}
$$

where

$$
\begin{aligned}
& H\left(p_{k+1}, x_{k}, u_{k}\right)=\left\langle p_{k+1}, f\left(x_{k}, u_{k}\right)\right\rangle-g\left(x_{k}, u_{k}\right), \\
& H\left(p_{k+1}, x_{k}, u_{k}^{*}\right)=\max _{u \in \Omega} H\left(p_{k+1}, x_{k}, u\right) .
\end{aligned}
$$

We can then obtain the discrete rigid body/ Moser Veselov equations as follows:

Proposition 5.2 Let $V=\sum_{k} \operatorname{Tr}\left(\Lambda U_{k}\right)$ where $\Lambda$ is a positive definite diagonal matrix and $U_{k} \in S O(n)$. The optimal control problem

$$
\min _{u_{k}} V
$$

subject to $Q_{k+1}=Q_{k} U_{k}$ for $Q_{k} \in S O(n)$, yields the optimal control equations

$$
\begin{aligned}
Q_{k+1} & =Q_{k} U_{k} \\
P_{k+1} & =P_{k} U_{k}
\end{aligned}
$$

where

$$
U_{k} \Lambda-\Lambda U_{k}^{T}=P_{k}^{T} Q_{k}-Q_{k}^{T} P_{k}
$$

For $M_{k}=Q_{k}^{T} P_{k}-P_{k}^{T} Q_{k}$ and $\Omega_{k}=Q_{k+1}^{T} Q_{k}=U_{k}^{T}$ we obtain the Moser Veselov equations

$$
M_{k+1}=\Omega_{k} M_{k} \Omega_{k}^{T}
$$

Note that $V=\sum_{k} \operatorname{Tr}\left(Q_{k} \Lambda Q_{k+1}\right)$, the Moser Veselov functional, but that the functional is linear in the controls. Note also that the discretization here is slightly different from that in the previous section.

\section{Parameterized Equations}

It is a remarkable fact that the dynamic rigid body equations on $S O(n)$ and indeed on any semisimple Lie group are integrable (Mishchenko and Fomenko [1976]). A key observation in this regard, due to Manakov, was that one could write the generalized rigid body equations as Lax equations with parameter:

$$
\frac{d}{d t}\left(M+\mu \Lambda^{2}\right)=\left[M+\mu \Lambda^{2}, \Omega+\mu \Lambda\right]
$$

The nontrivial coefficients of $\mu$ in the traces of the powers of $M+\mu \Lambda^{2}$ then yield the right number of independent integrals in involution to prove integrability of the flow on a generic adjoint orbit of $S O(n)$ (identified with the corresponding coadjoint orbit). Moser and Veselov [1991] show that there is a corresponding formulation of the discrete rigid body equations with parameter.

Our formulation of the rigid body equations, which treats configuration and momentum variables symmetrically, provides an approach to analyzing integrability of the full rigid body equations (kinematics and dynamics). Another approach may be found in Mischenko and Fomenko [1978].

It is possible in our setting also to write the full rigid body equations with parameter and thus to investigate integrability of the full flow and its discretized counterpart. We indicate briefly how to do this. Integrability will be discussed in full in a forthcoming paper. Let

$$
J(\Omega)=\Lambda \Omega+\Omega \Lambda
$$

be as in Section 2. We then consider the equations with parameter:

$$
\begin{gathered}
\dot{Q}_{\mu}=Q_{\mu}(\Omega+\mu \Lambda) \\
\dot{P}_{\mu}=P_{\mu}(\Omega+\mu \Lambda)
\end{gathered}
$$

Setting

$$
M_{\mu}=P_{\mu}^{-1} Q_{\mu}-Q_{\mu}^{-1} P_{\mu}
$$

we find

$$
\dot{M}_{\mu}=\left[M_{\mu}, \Omega+\mu \Lambda\right]
$$

A remarkable feature of the double bracket formulation discussed here is that it is particularly useful for analyzing the integrability of the full rigid body equations. As discussed above, the Manakov parameter formulation gives us the integrals on the orbit (for the dynamics). We may expand the number of integrals by including the spatial momenta as follows.

Consider the equation

$$
[\hat{P}, \hat{Q}]=\left[\begin{array}{ll}
Q P^{T}-P Q^{T} & 0 \\
0 & Q^{T} P-P^{T} Q
\end{array}\right] .
$$

In the left invariant formulation, the lower right block is the body momentum while the upper left block is (minus) the spatial momentum.

We can then write down integrals of the motion in a similar fashion to those we wrote down for biinvariant flows on Grassmannians in Bloch, Brockett and Ratiu [1997]. They group in a pleasing fashion into the Manakov integrals and the spatial momenta.

Consider the left invariant setting. To obtain the Manakov integrals set $\hat{J}=\operatorname{diag}(0, J)$ as before. The Manakov integrals are then given by

$$
\operatorname{Tr}([\hat{P}, \hat{Q}]+\mu \hat{J})^{k}
$$


The spatial momenta are given by

$$
\operatorname{Tr}\left(\pi_{U}([\hat{P}, \hat{Q}]) A\right)
$$

where $A$ runs through a basis of spatial momenta and $\pi_{U}$ is projection onto the upper left block.

Because of this upper/lower structure it is not hard to show involution of the spatial and body integrals. We will carry out the details in a forthcoming paper. In addition we will discuss integrability in the discrete case and its connection with the work Moser and Veselov [1991] and Deift, Li and Tomei [1992].

Acknowledgement: We would like to thank R. Brockett for useful discussions on this material.

\section{References}

Bloch, A. M. [1990] Steepest descent, linear programming and Hamiltonian flows. Contemp. Math. AMS 114, 77-88.

Bloch, A.M., R.W. Brockett and T.S. Ratiu [1992] Completely integrable gradient flows. Comm. Math. Phys. 147, 57-74.

Bloch, A.M., R. W. Brockett, and P.E. Crouch [1997] Double bracket equations and geodesic flows on symmetric spaces. Comm. Math Phys 187,357373.

Bloch, A.M. and P.E. Crouch [1994] Reduction of Euler Lagrange problems for constrained variational problems and relation with optimal control problems. Proc. CDC 33, IEEE, 2584-2590.

Bloch, A.M. and P.E. Crouch [1995] On the geometry of optimal control and geodesic flows Proc. $C D C$ 34, IEEE, 3283-3288.

Bloch, A. M. and P. E. Crouch [1996] Optimal control and geodesic flows Systems and Control Letters 28, 65-72.

Bloch, A.M., H. Flaschka, and T.S. Ratiu [1990] A convexity theorem for isospectral sets of Jacobi matrices in a compact Lie algebra. Duke Math. J. 61, 41-66.

Brockett, R.W. [1973] Lie theory and control systems defined on spheres. SIAM J. Appl. Math./ 23, 213-225.

Brockett, R.W. [1988] Dynamical systems that sort lists and solve linear programming problems. Proc. IEEE 27, 799-803 and Linear Algebra and its Appl. 146, (1991), 79-91.

Brockett, R.W. [1993] Differential geometry and the design of gradient algorithms. Proc. Symp. Pure Math., AMS 54, Part I, 69-92.
Brockett, R.W. [1994] The double bracket equation as a solution of a variational problem. Fields Institute Comm. 3, 69-76.

Crouch, P.E. and F. Silva Leite [1991] Geometry and the dynamic interpolation problem. Proc. A.C.C. Boston, 1131-1136.

Deift, P., P. Li and C. Tomei [1992] Loop groups, discrete versions of some classical integrable systems and rank 2 extensions, Memoirs of the AMS 100, No. 479, AMS, Providence.

Faybusovich, L.E. [1988] Explicitly solvable optimal control problems. Int. J. Control 48 No. 6, 235250 .

Helgason, S. [1978] Differential geometry, Lie groups, and symmetric spaces, Academic Press.

Marsden, J. and J. Wendlandt [1997] Systems with symmetry, variational principles and integration algorithms, Current and Future Directions in Applied Mathematics (M. Alber, B. Hu and J Rosenthal eds.), Birkhuaser.

Marsden, J., G. Patrick and S. Shkoller [1997] Multisymplectic Geometry, variational integrators, and nonlinear PDE's, Comm. Math. Phys. (to appear).

McLachlan, R. and C. Scovel [1995] Equivariant constrained symplectic integration, J. Nonlinear Science 5, 233-256.

Mischenko, A. and Fomenko A. [1976] The integrability of Euler equations on semisimple Lie algebras. Doklady Akad. Nauk CCCP 231, 536-538.

Mischenko, A. and Fomenko A. [1978] General Liouville method of integration of Hamiltonian systems. Functional analysis and its applications 12, 46-56.

Moser, J. and A. Veselov [1991] Discrete versions of some classical integrable systems and factorization of matrix polynomials Comm. Math. Phys 139, 217-243.

Ratiu, T. [1980] The motion of the free n-dimensional rigid body. Indiana U. Math. J., 29, 609-627.

Thimm, A. [1981] Integrable Hamiltonian systems on homogeneous spaces. Ergod. Th. and Dynam. Systems 1, 495-517.

Wendlandt, J.M. and J.E. Marsden [1997] Mechanical integrators derived from a discrete variational principle, Physica $D$ 106, 223-246. 